Review Article

DOI: $10.36959 / 584 / 458$

\title{
Balovaptan and Autism Spectrum Disorder
}

\author{
Diego Lopez da Silva ${ }^{1}$ (D) Lara Lídia Ventura Damasceno ${ }^{1}$, Ernandes Félix Sales ${ }^{1}$, Laís Kelly \\ Maciel Rabelo ${ }^{1}$, Maria Beatriz Nunes de Carvalho ${ }^{1}$, Talita Maria de Oliveira Rabelo ${ }^{1}$, Mayra \\ Larisse Teixeira Alexandre ${ }^{1}$, Jose Nilo Rodrigues da Silva ${ }^{1}$ and Gislei Frota Aragão $0^{1,2^{*}}$
}

${ }^{1}$ Health Sciences Center, Ceará State University, Fortaleza, Ceará, Brazil

${ }^{2}$ Drug Research and Development Center, Federal University of Ceará, Fortaleza, Ceará, Brazil

\begin{abstract}
Balovaptan, an antagonist of the vasopressin system, has been shown to be a drug with potential to treat the main symptoms of Autism Spectrum Disorder (ASD). The objective of this work was to gather and analyze information already published about the mechanism of action, clinical trials, dosage information, pharmacokinetics, and safety profile of balovaptan, bringing an update on the current situation of this molecule in ASD. A bibliographic review was conducted through the Embase, PubMed, Science Direct, LILACS, SciELO, Web of Science, Google Scholar, and Cochrane Library databases. Results showed that balovaptan is a potent, selective, and brain-penetrant V1a receptor antagonist that may improve social behaviors and communication. Balovaptan received the Breakthrough Therapy designation from the US Food and Drug Administration. Balovaptan showed a favorable safety profile and good pharmacokinetic parameters in studies using doses that varied from 1.5 to $10 \mathrm{mg}$ orally. Although preliminary results have shown promise, the balovaptan study was terminated because improvement in the core symptoms of ASD was lower than expected. However, further studies with substitute molecules should be encouraged, given the therapeutic potential of vasopressin axis modulation and its implications for social behavior.
\end{abstract}

\section{Keywords}

Central nervous system, Autism spectrum disorder, Drug evaluation, Balovaptan

\section{Introduction}

Vaptans are a class of drugs composed of vasopressin receptor antagonists. Vasopressin, also called antidiuretic hormone (ADH), is an endogenous neuropeptide produced in the paraventricular and supraoptic nuclei of the hypothalamus that can act on $\mathrm{V} 2$ receptors, expressed on the kidney, promoting water resorption, and on $\mathrm{V} 1 \mathrm{a}$ and $\mathrm{V} 1 \mathrm{~b}$ receptors, found in various tissues such as muscles, vessels, uterus, and central nervous system [1,2].

Overall, vasopressin plays an important role in circulatory homeostasis of sodium and regulation of osmolality. The activation of its receptors on vascular smooth muscle cells and myocardium results in vasoconstriction, increased afterload, and hypertrophy. In the kidneys, especially in the renal tubules, the action on these receptors results in free water absorption. Therefore, the use of vasopressin antagonists results in vasodilation and water excretion, wen antagonizing V2 receptors [3].

Studies in primates have shown the distribution of $\mathrm{V} 1 \mathrm{a}$ receptors in several regions of the central nervous system, such as: Lateral septum, basal ganglia, suprachiasmatic nucleus, amygdala, neocortex, olfactory system, and ventromedial nucleus of the hypothalamus. It is speculated that this distri- bution of receptors allows them to relate to the processing of multimodal signals, which strengthens the hypothesis of their participation in higher mental processes, such as memory. In addition, the modulation of these circuits may be related to the genesis of some disorders, such as depression, anxiety, and aggressive behavior [4-6].

Balovaptan (Figure 1) is a vasopressin receptor antagonist and is currently being developed by Roche Pharmaceutical Company (Switzerland). Balovaptan, at first, showed to be a drug with innovative potential to treat the main symptoms of Autism Spectrum Disorder (ASD), seeing that V1a receptor antagonism is a potential therapeutic target for the resolu-

*Corresponding author: Dr. Gislei Frota Aragão, Health Sciences Center, Ceará State University; Drug Research and Development Center, Federal University of Ceará, Silas Munguba Avenue, 1700 - Itaperi, Fortaleza, Ceará, Brazil-60714-903, Tel: +55-(85)-98830-2494

Accepted: December 07, 2020

Published online: December 09, 2020

Citation: Silva DL, Damasceno LLV, Sales EF, et al. (2020) Balovaptan and Autism Spectrum Disorder. Insights Biomed Res 4(1):120-124 


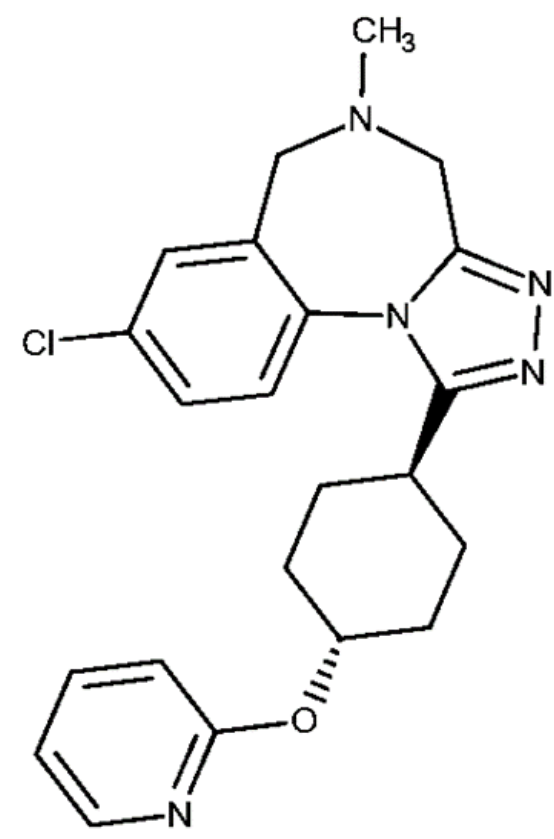

IUPAC Name: 12-chloro-8-methyl-3-[(1r,4r)-4-(pyridin-2-yloxy) cyclohexyl]-2,4,5,8-tetraazatricyclo[8.4.0.0^\{2,6\}]tetradeca1(14),3,5,10,12-pentaene.

Chemical Formula: C22H24CIN5O.

Mono mass: 409.1669381 (https://www.drugbank.ca/drugs/ DB14823)

Figure 1: Structure of Balovaptan.

tion of communication and social interaction deficits characteristic of ASD [7].

The aim of this study was to present an update about this new molecule, focusing on its mechanism of action, use profile, dosage information, pharmacokinetics, and safety profile. For this, the following databases were searched: EMBASE, PUBMED, LILACS, Science Direct, SciELO, Web of Science, Google Scholar, and Cochrane Library.

\section{Results and Discussion}

There are still few published studies on balovaptan, as it is a new drug that is under development by Roche Pharmaceutical Company. In January 2018, balovaptan received the Breakthrough Therapy designation from the US Food and Drug Administration (https://www.roche.com/investors/ updates/inv-update-2018-01-29.htm), known as an authorization for the development of drugs with early evidence of clinical benefits for patients without treatment options.

This designation was due to studies that showed improvements in core symptoms of ASD, such as communication and sociability deficits, with the use of balovaptan. This hypothesis came from the application in rat valproate models of ASD, in which, the capacity for socialization was increased after the use of balovaptan. This suggests, therefore, the existence of prosocial effects in the use of balovaptan. Thus, a possible relationship between the inhibition of V1a receptors and the improvement in communication and social behavior could be extrapolated to humans with ASD $[8,9]$.
This effect of balovaptan has a positive impact on scales such as the "Pediatric Quality of Life Inventory" (PedsQL), Generic Core Scale, Cognitive Functioning Scale, and Family Impact Module. These scales were used by Bolognani, et al. [8] to assess emotional, social, and school-related aspects of patients using balovaptan. After 12 weeks of using balovaptan, patients showed improvements in the scales, which suggests an increase in the quality of life of patients with ASD using balovaptan [8,9]. However, such studies showed lower-than-expected improvements and had their activities terminated, given the low probability of further changes in behavioral scales assessed by these studies in individuals with ASD, given the hypothesis of prosocial benefits of balovaptan. Modulation of the vasopressin pathway may produce potential benefits for individuals with ASD and other psychiatric disorders, as can be seen in the current landscape of papers, clinical trials, and ongoing researches with balovaptan and other vasopressin pathway modulators [10]. Some of the main characteristics of balovaptan will be discussed below.

\section{Mechanism of action}

The mechanism of action of balovaptan consists of a reversible interaction with $\mathrm{V} 1 \mathrm{a}$ receptors to antagonize the action of vasopressin, which prevents its functioning. V1a receptors in the central nervous system act by coupling to $G$ protein, which is classified into the subunits Gs, Gq11, and $\mathrm{Gi} / \mathrm{o}$. When coupled to $\mathrm{Gs}$, the receptor acts via adenylate cyclase activation, unlike Gi/o, which acts by inhibiting this enzyme. Gq11 acts via phospholipase $C$ activation and consequent modulation of intracellular calcium levels $[6,8]$.

The use of balovaptan is associated with reduced activation of some brain areas, such as: Hypothalamus, raphe nucleus, nucleus accumbens, hippocampus, piriform cortex, among others. The suppression of these areas may play a key role in the control of hyperactivated regions in ASD, which are known to compromise social behavior in these individuals. One of these regions is the prefrontal cortex (PFC), which is hyperactivated in patients with ASD, as measured by electroencephalography, and has been correlated with the core symptoms of ASD. It is postulated that the modulation of these nuclei with a high density of V1a receptors may, through ascending projections, reduce the activity of the PFC and of other regions to which it is connected, such as the habenula, to then have prosocial effects in these individuals $[11,12]$.

This inhibitory effect alters the levels of vasopressin and consequently of its receptors. Thus, the neuromodulatory effects developed by the vasopressin axis, such as those related to the limbic system and PFC, are regulated. This control over certain neuronal pathways allows for possible manipulation of brain areas related to the core symptoms of ASD, as is the case with the limbic system and PFC and its relationship with social behavior $[6,8]$.

\section{Clinical trials}

Balovaptan phase II clinical trial (VANILLA-Vasopressin Antagonist to Improve sociaL communication in Autism) was performed in individuals who met the criteria for ASD as defined by the Diagnostic and Statistical Manual of Mental Dis- 
orders (DSM-5) and International Classification of Diseases (ICD-10). Individuals should also have adequate scores on the Clinical Global Impression-Severity (CGI-S) scale, Social Response Scale-2 (SRS-2) Vineland-II Adaptive Behavior Scales (VINELAND-II), Autism Diagnostic Observation Schedule (ADOS), and Aberrant Behavior Checklist (ABC) [8].

The inclusion criteria used by Bolognani, et al. [8] were: Good general health, defined as the absence of evidence of any active or chronic disease - such as hypertension, cardiovascular disease, diabetes, or other endocrine disorders, Body Mass Index (BMI) between 18 to $40 \mathrm{~kg} / \mathrm{m}^{2}$, Intelligence Quotient $(I Q) \geq 70$, preserved language, vision, and hearing, no history of head injury, other neurological disorders, or psychiatric illness, in addition to not being an alcohol or drug abuser [8].

Healthy status is assessed by performing a detailed medical and surgical history, along with complete physical examination including vital signs, 12-lead electrocardiogram, hematology, blood chemistry, urinalysis, serology, and BMI [8].

The sensory system preservation requirement is explained by the need to measure improvement in visual scanning, abnormal eye gaze patterns, and identification of smells and emotions. This occurs because these mechanisms are linked to the positive effects associated with balovaptan use, related to primitive behaviors and aspects of the information acquisition process, serving as an indication of efficacy $[7,13]$.

Participation of individuals with clinically significant and unstable psychotic affective disorders, substance use disorders, suicidal ideation, uncontrolled epilepsy, decompensated cardiovascular disease or hypertension, syncope episodes, positive serological results for hepatitis $\mathrm{B}, \mathrm{C}$, or HIV, concomitant disease or condition that could interfere or whose treatment could interfere with the conduct of the study, hematologic abnormalities, coagulopathies, and presence of malignancies, was discouraged [8].

The VANILLA study used only males, between 18 and 45 years, as subjects. This could be explained by the increased incidence of ASD in males, although there may be a difference in effects of $\mathrm{V} 1 \mathrm{a}$ receptor antagonism between men and women. In males, balovaptan affects primarily conciliatory behaviors, whereas in females, it affects areas playing a key role in reward, social bonding, and memory. However, it is uncertain that this is the main reason why the study was conducted with only a male population $[8,14,15]$.

In the VANILLA study, dose-dependent, significant, and clinically meaningful improvements on the Vineland-II composite score were observed for participants treated with balovaptan. This was due to improvements in socialization and communication domains [16].

Another clinical trial testing balovaptan is the V1ADUCT study (https://clinicaltrials.gov/ct2/show/NCT03504917). It is a phase III, randomized, double-blind, placebo-controlled, efficacy, and safety study of balovaptan in male e female adults with ASD, with a 2-year open-label extension. It is an interventional clinical trial, and the target enrolment was approximately 350 participants. V1ADUCT aims to evaluate efficacy, safety, and pharmacokinetics of $10 \mathrm{mg}$ of oral administration balovaptan once a day compared with matching placebo in adults with ASD.

The primary outcome of the study consists of observing changes in the Vineland Adaptative Behavior Scales (Vineland-II) two-domain composite (2DC) score from the $24^{\text {th }}$ week of intervention with balovaptan, with a focus on the effectiveness of the therapy. However, the study was terminated due to futility analyses, that is, preliminary results were below the primary objective proposed for the study. Therefore, it is unlikely that, at the end of the tests, satisfactory changes in the VINELAND - II 2DC score will be found.

Another Phase II multi-center, randomized, double-blind, 3-arm, parallel group study, known as aV1ation, targeting a population of approximately 350 individuals between 5 and 17 years with ASD, was terminated. The primary objective of the test was to investigate the safety, efficacy, and pharmacokinetics of balovaptan at doses of $4 \mathrm{mg}$ or $10 \mathrm{mg}$ compared to placebo. Patients were assessed for 24 weeks to verify, at the end of this period, changes in the Vineland Adaptative Behavior Scales (Vineland-II) two-domain composite (2DC) score and other scores. The study was terminated due to a lack of clinical or statistical benefits over the proposed primary outcome. (https://clinicaltrials.gov/ct2/show/NCT02901431).

\section{Dosage approaches}

Bolognani, et al. [8] used three different dosages of balovaptan, which were administered orally for a period of 12 weeks. The $1.5 \mathrm{mg}$ dose was initially given to measure and evaluate drug parameters and pharmacokinetic behavior. However, the $4 \mathrm{mg}$ and $10 \mathrm{mg}$ doses, when compared to placebo, showed a significant clinical improvement regarding socialization and communication, thus suggesting a possible effectiveness of both doses. Complementarily, Squassante, et al. [9] also performed similar analyses, with the same doses to evaluate the safety and efficacy in male adults with ASD for the treatment of social and communication deficits, with a view to health-related quality of life in these patients.

In addition, the V1ADUCT study, developed by the Hoffmann-La Roche Laboratory (2019), was designed to evaluate the efficacy, safety and pharmacokinetics of balovaptan. The study used adults (18 years and older) with ASD to compare the effects of a $10 \mathrm{mg}$ dose of balovaptan once daily with placebo. However, the final results of the study were not found.

\section{Pharmacokinetics}

Balovaptan belongs to the vaptan class and acts as a highly selective competitive antagonist for $\mathrm{V} 1 \mathrm{a}$ receptors present in the central nervous system. Balovaptan is administered orally, with rapid absorption by the gastrointestinal tract, reaching a peak plasma concentration in 3 hours. Balovaptan half-life is approximately 47 hours and its metabolism is mainly hepatic, since it is a vaptan. The maximum mean concentration found in patients after the second week of treatment varies according to the initial dosage. Individuals who received $1.5 \mathrm{mg}, 4$ $\mathrm{mg}$, and $10 \mathrm{mg}$ had maximum plasma concentrations of 8,20 and 60 nanograms/milliliter, respectively $[8,17]$. 
In addition, mathematical models based on the pharmacokinetic and pharmacodynamic characteristics of balovaptan, associated with ex vivo tests, estimated that the occupancy rate of V1a receptors was more than $80 \%$ with the administration of $4 \mathrm{mg}$, and more than $90 \%$ with the administration of $10 \mathrm{mg}$, suggesting strong selectivity of balovaptan. Food was shown to have no relevant effect on the pharmacokinetics of balovaptan [8].

\section{Safety profile}

Despite being a drug still in the testing phase, balovaptan already shows some evidence of a satisfactory safety profile, despite the need for further studies to rule out any adversity not yet identified [8]. Following are some points about the safety of balovaptan.

Adverse events: The VANILLA trial found some adverse events in adults undergoing the study. The prevalence of these events varied between the different doses used. Adverse events were reported in $64 \%$ of the placebo group and in $78.1,66.2$, and $66.7 \%$ of the balovaptan $1.5 \mathrm{mg}, 4 \mathrm{mg}$, and 10 mg-treated participants, respectively [8]. The most common adverse event was headache, with a frequency ranging from $12.5 \%$ to $13 \%$ in patients receiving balovaptan treatment, depending on the dosage used. Regarding serious adverse events, cases of rhabdomyolysis, acute psychosis, agitation, and sinus node dysfunction were observed during the study. However, only rhabdomyolysis and irritability were related to the use of balovaptan $1.5 \mathrm{mg}$ and $4 \mathrm{mg}$, respectively. None of the other serious adverse events were considered related to balovaptan treatment. No balovaptan-related changes in urinary, hematological, and electrocardiographic analyses were found. Thus, a reliable safety profile for adult men using balovaptan can be conjectured. However, further studies are needed to extrapolate the safety of balovaptan to other groups, such as children, adolescents, and women [8]. Table 1 summarizes the main side effects, the related dose, and their prevalence.

Drug interactions: No drug interactions were identified for the use of balovaptan. However, since balovaptan belongs to the vaptan class, its metabolism is through the cytochrome $P$ (CYP) 450 pathway, more specifically the subtype CYP450 $3 A 4$. Thus, drugs that inhibit the functioning of these enzymes (macrolides, diltiazem, and ketoconazole) can increase the plasma concentrations of vaptans, including balovaptan, and increase their antagonistic effect on V1a receptors. In situations where the activity of the CYP3A4 enzyme system is increased, e.g., when rifampicin and barbiturate are used, there may be a need for dose readjustment, since the degradation of vaptans will be increased, which reduces their plasma concentration [17].

Toxicity: No balovaptan-associated safety signals in terms of toxicity in skeletal muscle or in other systems were identified [8]. However, further studies should be conducted to establish a complete toxicity profile for balovaptan.

\section{Final Considerations}

Balovaptan is a molecule belonging to the vaptan class that potently and selectively antagonizes $\mathrm{V} 1 \mathrm{a}$ receptors present in the central nervous system. This inhibitory effect of the vasopressin pathway has been shown to have prosocial effects in adults with ASD, thus addressing the core symptoms of ASD. The dosage proposed as therapy varies from $1.5 \mathrm{mg}$ to $10 \mathrm{mg}$, administered orally and daily. Studies with this dosage suggest a satisfactory safety profile in adults with ASD. However, in the phase III study, the prosocial effects of balovaptan in adults with ASD were modest and did not demonstrate significant clinical changes, which led to the termination of clinical trials with balovaptan.

The modest results of balovaptan demonstrate the difference in behavior of ASD therapies in humans when compared to behavior in animals. This may be related to some elements, such as biological and behavioral differences between humans and animals. Another possible explanation is the multifactorial character of ASD, whose different elements constitute its complex pathophysiology, ranging from genetic to environmental aspects [10].

Thus, the modulation of a given pathway, such as the vasopressin axis, may not be sufficient to resolve the core symptoms of ASD and, perhaps, explains the difference in presentation between in vitro and animal treatments and human treatments. However, further studies should be encouraged to assess the apparent potential of vasopressin axis modulation, in addition to defining an extended safety profile for other population groups, such as children, women, and adolescents.

Table 1: The incidence of adverse events reported in more than $5 \%$ of participants and the doses at which they occurred [8].

\begin{tabular}{|l|l|l|l|l|}
\hline Adverse Effect & Placebo & Balovaptan 1.5 $\mathbf{~ m g}$ & Balovaptan 4 $\mathbf{~ m g}$ & Balovaptan 10 mg \\
\hline Headache & $\mathbf{n = 3 2}$ & $\mathbf{n = 7 7}$ & $\mathbf{n = 3 9}$ \\
\hline Anxiety & $21.3 \%$ & $12.5 \%$ & $13 \%$ & $12.8 \%$ \\
\hline Fatigue & $8 \%$ & $6.3 \%$ & 0 & $10.3 \%$ \\
\hline Abdominal discomfort & $2.7 \%$ & $6.3 \%$ & $1.3 \%$ & $10.3 \%$ \\
\hline Upper airway infection & $5.3 \%$ & 0 & $2.6 \%$ & $10.3 \%$ \\
\hline Weight gain & $2.7 \%$ & $3.1 \%$ & $9.1 \%$ & $5.1 \%$ \\
\hline Nasopharyngitis & $4 \%$ & $12.5 \%$ & $5.2 \%$ & 0 \\
\hline Irritability & $5.3 \%$ & $9.4 \%$ & $5.2 \%$ & 0 \\
\hline
\end{tabular}


Although the balovaptan study did not achieve the expected success, the hypothesis of vasopressin axis modulation and its implications for the central nervous system is a target for new molecules, including the class of vaptans, to be studied in the future for the treatment of ASD.

\section{Conflict of Interest}

All authors declare that there is no conflict of interest.

\section{Acknowledgement} work.

We thank Ms Livia Condah Kaghofer for translating this

\section{References}

1. Filipatos $T$, Elisaf M, Liamis G (2019) Pharmacological management of hyponatremia. Expert Opinion on Pharmacotherapy 19: 1337-1334.

2. Insel TR (2010) The challenge of translation in social neuroscience: A review of oxytocin, vasopressin, and affiliative behavior. Neuron 65: 768-779.

3. Verbalis JG (2010) Vasopressin V2 receptor antagonists. J Mol Endocrinol 29: 1-9.

4. Benarroch EE (2013) Oxytocin and vasopressina: Social neuropeptides with complex neuromodulatory functions. Neurology 80: 1521-1528.

5. Young, Toloczko, Insel (1999) Localization of vasopressin (V1a) receptor binding and mRNA in the rhesus monkey brain. Journal of Neuroendocrinology 11: 291-297.

6. Stoop R (2012) Neuromodulation by oxytocin and vasopressin. Neuron 76: 142-159.

7. Chadman KK, Fernandes S, DiLiberto E, et al. (2019) Do animal models hold value in Autism spectrum disorder (ASD) drug discovery? Taylor \& Francis Online 14: 727-734.
8. Bolognani F, Rubido MV, Squassante L, et al. (2019) A phase 2 clinical trial of a vasopressin $\mathrm{V} 1$ a receptor antagonist shows improved adaptive behaviors in men with autism spectrum disorder. Sci Transl Med 11.

9. Squassante L, Bolognani F, Smith J, et al. (2018) Effects of balovaptan on health related quality of life of adult men with ASD: Results from a phase 2 randomized double-blind placebo-controlled study (vanilla). J Am Acad Child Adolesc Psychiatry 57: S231.

10. D'arc BF, Mottron L, Elsabbagh M, et al. (2019) Tinkering with the vasopressin pathway in autism. Transl Med 11.

11. Schnider P, Bissantz C, Bruns A, et al. (2020) Discovery of balovaptan, a vasopressin 1a receptor antagonist for the treatment of autism spectrum disorder. J Med Chem 63: 1511-1525.

12. Benekareddy M, Stachniak TJ, Bruns A, et al. (2018) Identification of a corticohabenular circuit regulating socially directed behavior. Biol Psychiatry 83: 607-617.

13. Umbricht D, Del Valle RM, Hollander E, et al. (2017) A single dose, randomized, controlled proof-of-mechanism study of a novel vasopressin 1a receptor antagonist (RG7713) in high-functioning adults with autism spectrum disorder. Neuropsychopharmacology 42: 1914-1923.

14. Rilling JK, DeMarco AC, Hackett PD, et al. (2012) Effects of intranasal oxytocin and vasopressin on cooperative behavior and associated brain activity in men. Psychoneuroendocrinology 37 : 447-461.

15. Rilling JK, DeMarco AC, Hackett PD, et al. (2014) Sex differences in the neural and behavioral response to intranasal oxytocin and vasopressin during human social interaction. Psychoneuroendocrinology 39: 237-248.

16. Sanders K (2019) Evaluating balovaptan for the core symptoms of autism using vineland ${ }^{\mathrm{TM}}$-II adaptive behavior scales: Present experience and future directions. Panel Presentation. Palais des congres de Montreal, Montreal, Québec, Canada.

17. Villabona C (2010) Antagonistas del receptor de vasopresina: Los vaptanes. Endocrinología y Nutrición 57: 41-52.

DOI: $10.36959 / 584 / 458$

Copyright: (c) 2020 Silva DL, et al. This is an open-access article distributed under the terms of the Creative Commons Attribution License, which permits unrestricted use, distribution, and reproduction in any medium, provided the original author and source are credited. 